\section{Hacia la Implantación del Concepto de "Merchandise Management" como Herramienta Efectiva para el Manejo de Mercancía en la Industria de Venta de Alimentos al Detal en Puerto Rico}

\author{
José Antonio Quevedo
}

El método de "Merchandise Management" le permite al detallista administrar estratégicamente su empresa. Este proceso descubre un nuevo concepto organizacional que, más allá de ser un método tradicional de toma de inventario, dicta la integración de responsabilidades y decisiones en las áreas de mercadeo, compra, venta y manejo de mercancía. Esta integración es esencial para permitir a gerentes asignar efectivamente inventario de productos y manejar el espaciơde almacén disponible. Es precisamente esta responsabilidad integrada a las áreas aquí mencionadas, en unión a un proceso de planificación estratégica de categoría de productos, lo que diferenciará en el futuro el proceso de "Merchandise Management" de otros enfoques y métodos tradicionales de compra y manejo de mercancía.

El propósito de este estudio es determinar la posibilidad de implantar este sistema, así como ver los beneficios específicos que obtendrá el detallista de alimentos en Puerto Rico. En específico se intenta medir el nivel de conocimiento de los empresarios locales de la industria de venta de alimentos al detal sobre este concepto y los beneficios que ofrecerá la implantación del mismo para sus empresas; y medir las actitudes hacia la adopción de este concepto por parte de las personas que dirigen las principales empresas locales de venta de alimentos al detal con el fin de acelerar el proceso de adopción del mismo.

Los principales hallazgos de la investigación indican que los detallistas de alimentos en P.R. tienen, en general, una actitud favorable hacia la implantación de este concepto en sus respectivas empresas. No obstante, el investigador logró identificar algunas de las barreras en el pro- ceso de adopción, entre ellas: la complejidad en su implantación y en el mismo concepto, necesidad de personal capacitado, falta de experiencia, falta de conocimiento sobre su uso y sus beneficios, inversión de dinero que requiere la implantación del mismo, y resistencia (miedo) al cambio.

\section{La Utilidad del Estado de Flujos de Efectivo para Inversionistas y Acreedores}

\section{Ivelisse Céspedes}

La Junta de Normas de Contabilidad Financiera (FASB) emitió el Pronunciamiento 95, Estado de Flujo de Efectivo, en 1987, con el propósito de reemplazar el Estado de Cambios en la Posición Financiera. Este Pronunciamiento es la base de este estudio, cuyo propósito principal es determinar si el Estado de Flujo de Efectivo es útil para dos de sus principales usuarios: los inversionistas y los acreedores. Además, se determinará qué tipo de información en dicho Estado es útil para esos usuarios; qué decisiones toman con la información que obtienen del Estado de Flujo de Efectivo y cuál método, el directo o el indirecto, prefieren. Finalmente, se comparan los resultados con investigaciones previas sobre el Estado de Cambios en la Posición Financiera.

Los resultados de la investigación muestran que el Estado de Flujo de Efectivo es muy útil, tanto para los inversionistas como para los acreedores. Este contiene información necesaria para los usuarios que no se obtiene de los otros estados financieros. Es además una herramienta en la toma de decisiones, aunque no es la principal; los usuarios encuestados mostraron preferencia por el método indirecto. Estos hallazgos son similares a los de investigaciones previas sobre el Estado de Cambios en la Posición Financiera, predecesor del Estado de Flujo de Efectivo. 


\section{Modelo para Determinar la Condición Financiera de las Cooperativas de Ahorro y Crédito Establecidas en la Región de San Juan}

\author{
Sandra I. Colón Rodríguez
}

Ante la necesidad que tienen los administradores de las cooperativas de ahorro y crédito de contar con una herramienta que dé a conocer la condición financiera de las mismas, se desarrolló, por medio de esta investigación, un modelo matemático. Con el fin de desarrollar el modelo, se utilizó el análisis discriminante. Mediante éste, se diseñó una ecuación compuesta por las variables que mejor diferenciaron a los grupos bajo estudio. Una vez se seleccionaron éstas, se hizo la debida prueba de hipótesis.

Con el propósito de simplificar el estudio, se incluyeron solamente las cooperativas comunales de ahorro y crédito de la Región de San Juan. La información financiera de cada cooperativa se obtuvo para un período de tres años consecutivos de operaciones.

Cada cooperativa se evaluó en los renglones de liquidez; lucratividad, solvencia y comportamiento de los activos. Mediante el modelo se clasificaron más del $80 \%$ de las cooperativas incluidas en el estudio.

Se encontró que el modelo es útil a un nivel de significancia $(\alpha)$ de 0.25 y que puede predecir hasta con dos años de anticipación la condición financiera de estas cooperativas. Finalmente, se concluye que la investigación fue capaz de desarrollar una herramienta con la cual este tipo de cooperativa puede conocer su condición financiera en cualquier momento.

\section{Estudio de Viabilidad para la Creación de un Complejo Turístico Recreativo en el Pueblo de Barranquitas}

\section{Marcos Fernández Avilés}

La mayoría de las grandes economías capitalistas mundiales se componen de pequeñas empresas que tratan de sobrevivir acaparando esos pequeños nichos de mercado que las grandes empresas no desean atender.
Esta investigación analizó la posibilidad de emprender una gestión empresarial para el desarrollo de un complejo turístico recreativo en el pueblo de Barranquitas. Los objetivos de este estudio fueron: el analizar cómo maximizar la oportunidad de establecer un negocio dedicado a la rama de la industria del turismo y convencer, con datos contundentes, a inversionistas e instituciones financieras, de la viabilidad del plan propuesto.

El dramático incremento del ingreso per cápita en el centro de la Isla, el ingreso por grupo familiar, el ingreso de la mujer trabajadora, y los cambios en la población de 1980 a 1990, indican el desarrollo económico de la zona. Por otro lado, el número de paradores creció de 1981 a 1993 , de un 3\% a un $7 \%$.

La demanda analizada por el investigador demuestra que existe potencial económico, en el pueblo de Barranquitas, para el desarrollo de un complejo turístico. El análisis de demanda refleja una condición de estabilidad de la industria que redunda en beneficio económico para los que deciden invertir en este campo. Los estados financieros proyectados a cinco años reflejan ganancias comenzando con $\$ 9,351$ en el primer año de operaciones y terminando con $\$ 115,641$ en el año 2000. Basado en la información que se obtuvo a través de todo el proceso, el investigador entiende que es una buena alternativa el invertir en el campo del turismo con miras a establecer un negocio de esta categoría. También se recomienda el considerar otras estrategias de diversificación, como lo es el dedicarse a zona recreativa totalmente.

Estudio sobre el Valor Predictivo de los Requisitos de Admisión de la Escuela Graduada de Administración de Empresas en el Éxito de los Estudiantes

\section{Wanda M. Mattei Ballester}

El propósito de esta investigación es determinar si los requisitos de ingreso a la Escuela Graduada de Administración de Empresas de la Universidad de Puerto Rico, Recinto de Río Piedras, (EGAE), tienen valor predictivo en el éxito de los estudiantes que se matriculan en la misma. 
El éxito se definió como la capacidad del estudiante para aprobar todos los cursos con un índice mínimo de 3.00 .

Los requisitos de admisión que se utilizaron como variables predictoras fueron: el índice académico de bachillerato y la puntuación de la prueba de Admisión a Estudios Graduados (PAEG). Otras variables predictoras fueron: el tipo de Bachillerato y la universidad de procedencia. Los datos para esta investigación se obtuvieron de los expedientes de los estudiantes que mantiene la administración de la EGAE. Se consideraron los estudiantes que ingresaron del 1984 al 1987.

Los datos indican que las variables que se emplean como criterio de admisión tienen muy poco valor predictor por sí solas. Sin embargo, la puntuación del PAEG parece ser la variable con mayor fuerza predictora para todos los grupos de estudiantes.

\section{Segmentación de Mercados para Destinos Vacacionales Tropicales}

\section{Maristella Aguiló $\quad$ •}

Mediante este estudio se identificó la importancia que los estudiantes de 1995 de la Escuela Graduada de Administración de Empresas de la Universidad de Puerto Rico, Recinto de Río Piedras, le otorgaron a los criterios evaluativos que señala la literatura como los considerados al decidir un destino vacacional en un lugar tropical, así como la importancia de dichos criterios en el contexto de turismo interno.

Se empleó un análisis de conglomerados para identificar los diferentes segmentos presentes en la muestra. Éstos se definieron de acuerdo a las variables demográficas y a la importancia que le asignaron a los criterios evaluativos en la selección de destinos turísticos tropicales. Se definieron tres segmentos.

El primer segmento consideró los siguientes criterios como importantes: la diversidad de actividades que se ofrecieron fuera del lugar de alojamiento, el nivel de contacto con la naturaleza y la calidad de los servicios. El segundo segmento se distinguió por la importancia que le otorgó a: la calidad de los servicios en el lugar de alojamiento, la acce- sibilidad desde el Area Metropolitana, la disponibilidad de restaurantes, y la diversidad de actividades dentro del lugar de alojamiento. La mayoría de la muestra de estos dos segmentos consistió, principalmente, de mujeres solteras entre 20 y 29 años de edad, estudiantes a tiempo parcial, y con un ingreso anual entre $\$ 31,000$ y $\$ 40,000$. El tercer grupo se caracterizó por no haber realizado turismo interno. Este grupo, que se componía mayormente de hombres solteros entre las edades de 30 a 39 años, con un ingreso anual de $\$ 31,000$ y $\$ 40,000$, comprendió el $29 \%$ de la muestra.

En general, los sujetos de la muestra han realizado turismo interno y han considerado a Puerto Rico como una alternativa al planificar sus vacaciones en un destino turístico tropical.

\section{Sistemas de Costos de Calidad: Como Medir y Controlar los Costos en los Sistemas de Manufactura}

\author{
Ennie R. Bonilla
}

Durante los últimos años, las empresas le han asignado mayor importancia a la calidad de los productos. Esto se debe a que la calidad guarda una estrecha relación con la productividad y las ganancias de las empresas. Los sistemas de control de costos de calidad pueden ayudar a la gerencia a mejorar la posición de la compañía en el mercado.

Este trabajo consiste, principalmente, en una revisión de la literatura relacionada con los sistemas de control de costos de calidad. También se analizan algunos de los aspectos sobresalientes de los sistemas de control de costos de calidad, la relación entre calidad y productividad, y cómo medir y controlar los costos de calidad.

Se determinó que la empresa debe diseñar sistemas de control de costos de calidad de acuerdo con sus objetivos principales. Debe estar claro que estos sistemas son sólo una herramienta que les ayudará a determinar ciertos pasos a seguir para mejorar la productividad, la calidad, $y$, por ende, las ganancias de la empresa. 